\title{
Labyrinthe
}

$8 \mid 2001$

Numéro 8

\section{LE G.E.N.E.P.I. Groupement Étudiant National d'Enseignement aux Personnes Incarcérées}

Entretien avec Bastien Lampire, chargé de communication au G.E.N.E.P.I. Propos recueillis par Laurence Marie.

\section{(2) OpenEdition}

\section{Journals}

Édition électronique

URL : http://journals.openedition.org/labyrinthe/873

DOI : $10.4000 /$ labyrinthe.873

ISSN : 1950-6031

Éditeur

Hermann

Édition imprimée

Date de publication : 1 janvier 2001

Pagination : 127-131

\section{Référence électronique}

"LE G.E.N.E.P.I. Groupement Étudiant National d'Enseignement aux Personnes Incarcérées », Labyrinthe [En ligne], 8 | 2001, mis en ligne le 05 juin 2005, consulté le 30 avril 2019. URL : http:// journals.openedition.org/labyrinthe/873 ; DOI : 10.4000/labyrinthe.873

Ce document a été généré automatiquement le 30 avril 2019.

Propriété intellectuelle 


\section{LE G.E.N.E.P.I. Groupement Étudiant}

\section{National d'Enseignement aux Personnes Incarcérées}

Entretien avec Bastien Lampire, chargé de communication au G.E.N.E.P.I. Propos recueillis par Laurence Marie.

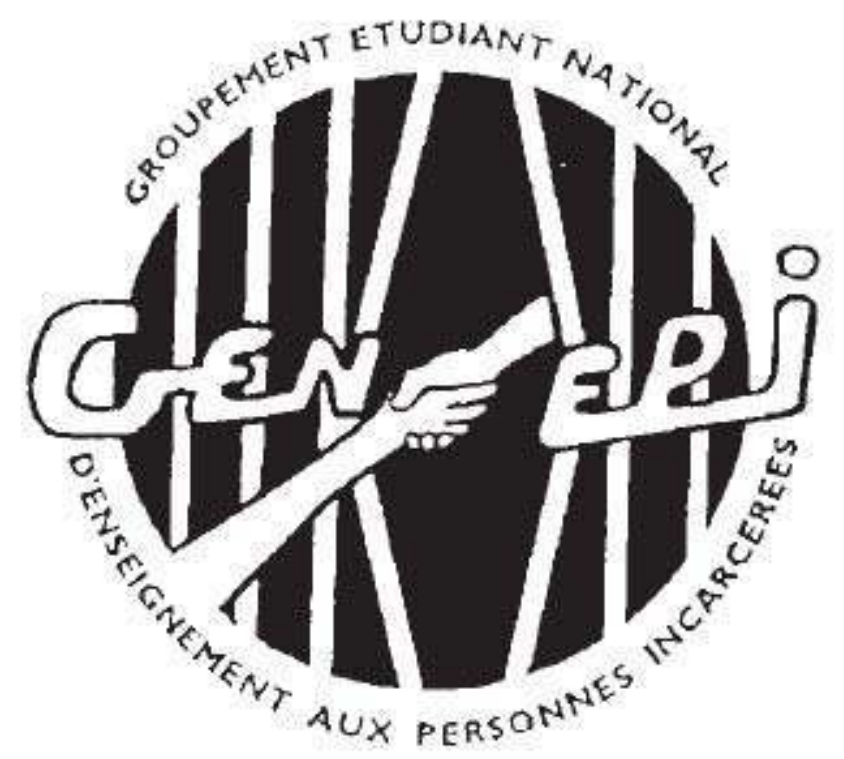

\section{Labyrinthe - Comment est néle G.E.N.EP.I. ?}

2 En 1974, des émeutes éclatent dans neuf prisons françaises. Six détenus trouvent la mort. Le Président de la République décide d'ouvrir le monde carcéral à l'opinion publique. C'est alors que naît l'idée de faire intervenir en prison de jeunes étudiants. En 1976, le Groupement Étudiant National d'Enseignement aux Personnes Incarcérées (G.E.N.E.P.I.) dépose ses statuts. Aujourd'hui, il regroupe 900 étudiants répartis en 47 groupes, intervenant dans 60 établissements pénitentiaires. 

?

Le G.E.N.E.P.I. était au départ totalement dépendant du Ministère de la Justice à qui il devait sa création. Mais à partir de 1981, le G.E.N.E.P.I. s'est opposé à la loi sûreté-sécurité et a coupé les ponts. Nous émettons des critiques quand elles nous semblent nécessaires. Mais nos relations avec la justice ne sont pas pour autant conflictuelles. Elles sont réglées par la convention G.E.N.E.P.I--Justice, qui précise ce que l'administration pénitentiaire doit accorder aux génépistes pour que leur intervention soit efficace. Mais parfois le personnel des prisons refuse d'appliquer ce que la convention stipule. Les surveillants sont en sous-effectif ( 38 pour 100 détenus, soit 13 pour 100 car ils fonctionnent par tiers), et parfois ils déplorent que l'association leur donne apparemment plus de travail à cause des allées et venues des intervenants.

6 Le G.E.N.E.P.I. a deux missions principales : l'intervention en détention ainsi que l'information et la sensibilisation du public. En détention, les membres du G.E.N.E.P.I. donnent des cours de soutien scolaire et aident à la préparation aux examens, en collaboration avec les instituteurs. Les résultats sont bons car les détenus sont très motivés et ils ont évidemment du temps. Les génépistes organisent aussi des activités socio-culturelles, à partir de leur projet personnel : musique, arts plastiques, revue de presse, code de la route, et même parfois équitation, VTT, à l'intérieur des murs. L'action Information et Sensibilisation du Public (I.S.P.) consiste en des expositions d'œuvres de détenus, des conférences, des "ciné-débats », organisés simultanément à l'intérieur des murs et à l'extérieur, parfois avec d'anciens détenus. Cette action a pour but de changer l'image négative que la société a de la prison. Car l'incarcération est une peine : la dette, une fois payée, doit être effacée. Le véritable enjeu de la détention est donc la réinsertion, mais le G.E.N.E.P.I. ne peut pas gérer cet aspect : il y a $70 \%$ de femmes dans l'association, et $96 \%$ d'hommes en détention. Vous imaginez le problème si un ancien détenu vient sonner chez une étudiante pour lui demander de l'héberger !

Labyrinthe - Qui sont les génépistes?

8 Avant tout des étudiants motivés. Ils interviennent entre 1 heure 30 et 2 heures par semaine de novembre à juin, quasiment sans interruption, car ils demandent aux détenus de ne jamais manquer l'activité à laquelle ils participent. De plus, les prisons sont souvent loin des centres-villes; et il faut préparer les activités... Les génépistes sont tous des étudiants : la moyenne d'âge est de 22 ou 23 ans. Ils viennent d'horizons très divers : de l'école du cirque à l'E.N.S. Beaucoup sont juristes, étudiants en psychologie. Ils signent pour un an, mais 70 \% reviennent l'année suivante. Et certains prennent même une carte d'étudiant juste pour pouvoir travailler dans l'association. Le G.E.N.E.P.I. donne un esprit d'ouverture et de tolérance. Il aide à se défaire des a priori négatifs sur les gens.

9 Labyrinthe - Et tout le monde peut adhérer?

10 Les demandes d'adhésion sont extrêmement nombreuses : le bouche à oreille fonctionne bien! Les candidats doivent donc assister à trois réunions, qui sont autant d'écrémages successifs : nous leur présentons le système carcéral, et l'association, avant l'entretien individuel final. Les étudiants qui veulent étudier les détenus pour leur mémoire de maitrise ou de thèse sont immédiatement refusés. Les détenus ne sont pas des cobayes.

11 Labyrinthe - Les génépistes ont-ils une formation spécifique? 
12 La formation est obligatoire. Une journée régionale de formation pédagogique aide les génépistes à lancer leur activité, avant la rencontre de professionnels du monde carcéral (surveillants, directeurs de prison, architectes...). Suit un week-end de formation régionale consistant en des débats sur un thème particulier : les victimes, les délinquants sexuels, la justice des mineurs... Le but est que les génépistes aient une culture sur la prison. Nous allons toujours boire un pot après les réunions pour pouvoir discuter ensemble de nos expériences respectives. Nos amis en ont souvent assez de nous entendre parler du G.E.N.E.P.I. !

13 Labyrinthe - Y a-t-il déjà eu des problèmes lors de l'intervention des génépistes en prison?

14 En 25 ans, il y a eu un gros problème tous les 5 ans environ : une génépiste est tombée amoureuse d'un détenu, un des membres a apporté du haschisch en détention. Mais nous prévenons le personnel que ce genre de choses peut se produire.

15 Labyrinthe - Que pensez-vous des récentes révélations de Véronique Vasseur, médecin-chef à la prison de la Santé?

16 Ce que raconte Véronique Vasseur dans son livre porte surtout sur les années 1993-1994. Elle signale seulement au détour d'une phrase la réforme de 1994 sur la santé en prison. L'administration pénitentiaire a beaucoup évolué depuis, même s'il est vrai que les conditions de détention sont mauvaises. En cas d'atteinte aux Droits de l'Homme, les Génépistes ont aussi un devoir de témoignage, stipulé dans la charte du G.E.N.E.P.I. Mais leurs interventions dans ce sens sont rares car ils ne passent jamais plus d'une demijournée en détention.

17 Labyrinthe-Comment est financée l'association?

18 Tous les membres sont bénévoles, sauf les représentants, qui sont tous détachés du contingent ou objecteurs de conscience. Même les femmes demandent à faire leur service national pour pouvoir se consacrer au G.E.N.E.P.I. : depuis 3 ans, ce sont elles qui ont été élues présidentes. Mais l'association est confrontée en ce moment à des problèmes de recrutement liés à la disparition du service national. Elle va devoir trouver un nouveau moyen de rémunérer ses représentants. Nous reportons nos espoirs sur le volontariat civil, mais aucun financement n'a été prévu pour l'instant. L'administration a récemment tiré parti des emplois-jeunes pour embaucher des agents de justice destinés à organiser des activités au sein de la prison, en concurrence avec les génépistes.

Labyrinthe - Comment voyez-vous l'avenir du G.E.N.EP.I. ?

20 Je suis pour la disparition de l'association. Son but est de participer à l'effort public en faveur de la réinsertion sociale des personnes incarcérées, et le personnel rémunéré par l'État, à travers les emplois-jeunes par exemple, pourrait remplir cette mission. Néanmoins, entre un détenu et un membre du personnel de l'administration, la relation est hiérarchique. Avec les membres du G.E.N.E.P.I., elle est égalitaire. Les génépistes ne connaissent pas le passé pénal des détenus, et ils ne veulent pas le connaître.

21 - La vocation du G.E.N.E.P.I. est de participer au décloisonnement de la prison en établissant un lien entre les détenus et le monde extérieur.

22 - Le G.E.N.E.P.I. est sans affiliation politique ni religieuse. Il est indépendant de toute institution quant à sa réflexion et ses prises de position.

23 - Le G.E.N.E.P.I., association citoyenne, attachée au respect des Droits de l'Homme, a le devoir de rendre compte de leurs violations éventuelles. 
24 - Le G.E.N.E.P.I. est constitué d'étudiants y exerçant leur citoyenneté. La Justice étant rendue notamment en leur nom, le G.E.N.E.P.I. se réserve le droit de faire part de ses réflexions auprès des citoyens et de leurs représentants.

25 - Le G.E.N.E.P.I. considère que toute peine doit nécessairement permettre la réinsertion dans la société. différents partenaires concernés. grande honnêteté.

- Le G.E.N.E.P.I. contribue à l'exercice du droit au savoir des détenus.

- Dans toutes ses activités, le G.E.N.E.P.I. est indifférent au passé pénal des détenus.

- La qualité des interventions du G.E.N.E.P.I. nécessite la plus grande concertation avec les

- Dans le cadre de l'information et de la sensibilisation du public, le

G.E.N.E.P.I. rappelle que son devoir de témoignage s'accompagne du souci de la plus

- La diversité de provenance des étudiants qui composent le

G.E.N.E.P.I. est un atout majeur de la qualité de son action.

- L'action du G.E.N.E.P.I. nécessite la formation des membres de l'association.

- L'action au sein du G.E.N.E.P.I. est indissociable d'une réflexion sur le système pénal et judiciaire.

- La réflexion sur l'action et la politique du G.E.N.E.P.I. doit être permanente et menée par ses membres. 